\title{
OPTIMAL STRUCTURAL DESIGN FOR GIVEN DEFLECTION UNDER PERIODIC LOADING*
}

\author{
BY \\ RAYMOND H. PLAUT \\ Brown University
}

Abstract. This paper is concerned with the minimum-weight design of structures subjected to periodic loading, under the constraint of a prescribed deflection at a specified point of the structure. For elastic sandwich beams, the method of stationary mutual potential energy is extended to cover periodic loading and a sufficient condition for stationary weight is derived.

1. Introduction. The optimal design of structures subjected to a single periodic concentrated load has been investigated by Icerman [1]. He obtained sufficient conditions for minimum-weight design under the constraint of a prescribed deflection at the point of application of the load. A generalization of this problem is considered in this paper in which the loading may consist of distributed loads, concentrated loads, and concentrated moments, all periodic with the same frequency, and the deflection is prescribed at any specified point of the structure.

The method to be used is different from that of [1]. The principle of stationary mutual potential energy, which was introduced by Shield and Prager [2] and has been applied to optimal structural design problems in [2]-[6], is extended here to cover the case of periodic loading. For elastic sandwich beams, a sufficient condition for stationary weight is derived from this principle. The cases of continuously varying stiffness and segmentwise constant stiffness are treated, and some examples are considered.

2. Stationary principle. Consider a beam that is simply supported, built in, or free at the ends $x=0$ and $x=l$, with variable bending stiffness $s(x)$ and mass per unit length $m(x)$. Denote the distributed load by $q(x)$ cos $\omega t$, typical concentrated loads by $Q$ $\cos \omega t$, and typical concentrated couples by $C \cos \omega t$. Let $u(x) \cos \omega t$ be the deflection of the beam. It is desired to find the design $s(x)$ which makes $\int m(x) d x$ stationary under the constraint $u\left(x_{0}\right)=u_{0}$, where $x_{0}$ is a specified cross-section of the beam and $u_{0}$ is a prescribed value.

Consider a second state of loading consisting of a single concentrated load $\bar{Q} \cos \omega t$ applied at $x=x_{0}$, with $\bar{u}(x) \cos \omega t$ the corresponding deflection of the beam. Let $u^{*}(x)$, $\bar{u}^{*}(x)$ be any kinematically admissible deflections. Then we define the mutual total energy as

$$
\begin{aligned}
U\left[u^{*}, \bar{u}^{*}, u, \bar{u} ; s\right]=\frac{1}{2}\left\{\int s u^{* \prime \prime} \bar{u}^{* \prime \prime} d\right. & -\int q \bar{u}^{*} d x-\sum Q \bar{u}^{*}-\sum C \bar{u}^{* \prime} \\
& \left.-\bar{Q} u_{0}-\omega^{2} \int m u \bar{u}^{*} d x-\omega^{2} \int m \bar{u} u^{*} d x\right\} .
\end{aligned}
$$

\footnotetext{
* Received April 14, 1970. Sponsored by the U. S. Army Research Office-Durham.
} 
With the use of the principle of virtual work combined with D'Alembert's principle [7] and following the procedure of [2], one can show that

$$
U\left[u^{*}, \bar{u}^{*}, u, \bar{u} ; s\right]-U[u, \bar{u} ; s]=\frac{1}{2} \int s\left(u^{* \prime \prime}-u^{\prime \prime}\right)\left(\bar{u}^{* \prime \prime}-\bar{u}^{\prime \prime}\right) d x
$$

where $U[u, \bar{u} ; s] \equiv U[u, \bar{u}, u, \bar{u} ; s]$. Therefore the mutual total energy is stationary at $u^{*}=u, \bar{u}^{*}=\bar{u}$.

Now consider a second design with bending stiffness $\hat{s}(x)$ and mass per unit length $\hat{m}(x)$, for which the deflection $\hat{u}(x)$ cos $\omega t$ under the loads $q, Q, C$ also satisfies the constraint $\hat{u}\left(x_{0}\right)=u_{0}$. The deflection caused by the single load $\bar{Q} \cos \omega t$ is designated as $\bar{u}(x)$ cos $\omega t$. Continuing the procedure of [2], one obtains the relation

$$
U[\hat{u}, \hat{u} ; \hat{s}]=U[u, \bar{u} ; s]+\frac{1}{2} \omega^{2}\left\{\int m u \bar{u} d x-\int \hat{m} \hat{u} \hat{u} d x\right\}
$$

and, finally,

$$
\begin{aligned}
& \int\left[(\hat{s}-s) u^{\prime \prime} \bar{u}^{\prime \prime}-\omega^{2}(\hat{m}-m) u \bar{u}\right] d x \\
&=\int\left[\hat{s}\left(u^{\prime \prime}-\hat{u}^{\prime \prime}\right)\left(\bar{u}^{\prime \prime}-\hat{u}^{\prime \prime}\right)-\omega^{2} \hat{m}(u-\hat{u})(\bar{u}-\bar{u})\right] d x .
\end{aligned}
$$

For designs $\hat{s}(x)$ in the neighborhood of the design $s(x)$, the right side of (2) vanishes to first order.

3. Optimal design. The beam is assumed to have a sandwich cross-section with a core of constant height, so that the mass-stiffness relation is given by

$$
m(x)=a^{2}+b^{2} s(x)
$$

where $a$ and $b$ are constants. If the deflections $u(x)$ and $\bar{u}(x)$ satisfy the optimality condition

$$
u^{\prime \prime}(x) \bar{u}^{\prime \prime}(x)-\omega^{2} b^{2} u(x) \bar{u}(x)=k^{2}
$$

where $k$ is a constant, then (2) becomes

$$
\int(\hat{m}-m) d x=\left(b^{2} / k^{2}\right) \int\left[\hat{s}\left(u^{\prime \prime}-\hat{u}^{\prime \prime}\right)\left(\bar{u}^{\prime \prime}-\bar{u}^{\prime \prime \prime}\right)-\omega^{2} \hat{m}(u-\hat{u})(\bar{u}-\bar{u} \hat{)})\right] d x
$$

and the structural weight of design $s$ is stationary with respect to the weight of all designs $\hat{s}$ in the neighborhood of $s$ for which the deflection constraint is satisfied. The design $s$ provides a local minimum if the right side of (4) is positive for nearby designs $\hat{s}$ and a global minimum if it is positive for all possible designs $\hat{s}$.

The optimality condition obtained in [1] is found to be a special case of (3). If the loading $q, Q, C$ consists only of a single load $Q \cos \omega t$ at $x=x_{0}$, then $\bar{u}=u$ if $\bar{Q}$ is set equal to $Q$ and (3) yields

$$
u^{\prime \prime 2}(x)-\omega^{2} b^{2} u^{2}(x)=k^{2}
$$

Eq. (4) takes the form

$$
\int(\hat{m}-m) d x=\left(b^{2} / k^{2}\right) \int\left[\hat{s}\left(u^{\prime \prime}-\hat{u}^{\prime \prime}\right)^{2}-\omega^{2} \hat{m}(u-\hat{u})^{2}\right] d x .
$$


For this special case, therefore, a sufficient condition for $\int \hat{m} d x>\int m d x$ is that (5) be satisfied and $\omega$ be less than the fundamental frequency of the beam having stiffness $\hat{s}(x)$.

Another special case is that of a beam with segmentwise constant stiffness. If the subscript $i$ refers to quantities in the $i$ th segment, then one obtains from (2)

$$
\sum_{i}\left\{\left(\hat{m}_{i}-m_{i}\right) \int\left(u_{i}^{\prime \prime} \bar{u}_{i}^{\prime \prime}-\omega^{2} b^{2} u_{i} \bar{u}_{i}\right) d x_{i}\right\}=0
$$

to first order, where $m_{i}=a^{2}+b^{2} s_{i}$. The weight of the beam for design $s_{i}$ is $\sum m_{i} l_{i}$, and it follows that a sufficient condition for stationary weight is

$$
\frac{1}{l_{i}} \int\left(u_{i}^{\prime \prime} \bar{u}_{i}^{\prime \prime}-\omega^{2} b^{2} u_{i} \bar{u}_{i}\right) d x_{i}=k^{2} \quad \text { (independent of } i \text { ). }
$$

In general the optimality conditions (3) and (7) are not simple to apply. For beams of continuously varying stiffness the optimality condition (3), the equation of motion of the beam under loads $q, Q, C$, and the equation of motion under $\bar{Q}$ form a system of three coupled differential equations in $s(x), u(x)$, and $\bar{u}(x)$. The situation is a little more hopeful in the case of segmentwise constant stiffness, since the equations of motion may be solved for $u_{i}(x)$ and $\bar{u}_{i}(x)$ in terms of the stiffnesses $s_{i}$. Substitution of the resulting deflections into the optimality condition (7) then yields the solution $s_{i}$ for stationary weight.

4. Examples. (A) Consider a cantilevered sandwich beam of length $l$ under a distributed load $q(x)$ cos $\omega t$. The beam is to be designed for minimum weight subject to the constraint that the deflection at the free end is to have the amplitude $u_{0}$.

Let $x=0$ at the built-in end and $x=l$ at the free end. The equation of motion for $u(x)$ is given by

$$
\left[s(x) u^{\prime \prime}(x)\right]^{\prime \prime}-\omega^{2} m(x) u(x)=q(x),
$$

with boundary conditions

$$
u(0)=0, \quad u^{\prime}(0)=0, \quad s(l) u^{\prime \prime}(l)=0, \quad\left[s(x) u^{\prime \prime}(x)\right]_{x=2}^{\prime}=0 .
$$

The deflection $\bar{u}(x)$ is caused by a concentrated load $\bar{Q} \cos \omega t$ acting on the beam at $x=l$, and hence

$$
\begin{gathered}
{\left[s(x) \bar{u}^{\prime \prime}(x)\right]^{\prime \prime}-\omega^{2} m(x) \bar{u}(x)=0,} \\
\bar{u}(0)=0, \quad \bar{u}^{\prime}(0)=0, \quad s(l) \bar{u}^{\prime \prime}(l)=0, \quad\left[s(x) \bar{u}^{\prime \prime}(x)\right]_{x-l}^{\prime}=-\bar{Q} .
\end{gathered}
$$

A sufficient condition for stationary weight is given by (3). To find the optimal design $s(x)$, the coupled equations (3), (S), and (9) must be solved together with their boundary conditions and the constraint condition $u(l)=u_{0}$.

(B) Again consider the cantilevered sandwich beam described above, but this time the bending stiffness is to have a constant value $s_{1}$ for $0 \leq x<c$ and a constant value $s_{2}$ for $c<x \leq l$. Denote $u(x) \equiv u_{1}(x)$ for $0 \leq x \leq c$ and $u(x) \equiv u_{2}(x)$ for $c \leq x \leq l$. Then (S) takes the form

$$
s_{i} u_{i}^{\prime \prime \prime}(x)-\omega^{2} m_{i} u_{i}(x)=q(x), \quad i=1,2,
$$

with boundary conditions 


$$
\begin{gathered}
u_{1}(0)=0, \quad u_{1}^{\prime}(0)=0, \quad u_{1}(c)=u_{2}(c), \quad u_{1}^{\prime}(c)=u_{2}^{\prime}(c), \\
s_{1} u_{1}^{\prime \prime}(c)=s_{2} u_{2}^{\prime \prime}(c), \quad s_{1} u_{1}^{\prime \prime \prime}(c)=s_{2} u_{2}^{\prime \prime \prime}(c), \quad s_{2} u_{2}^{\prime \prime}(l)=0, \quad s_{2} u_{2}^{\prime \prime \prime}(l)=0 .
\end{gathered}
$$

Similarly, if $\bar{u}(x) \equiv \bar{u}_{1}(x)$ for $0 \leq x \leq c$ and $\bar{u}(x) \equiv \bar{u}_{2}(x)$ for $c \leq x \leq l$, then

$$
s_{i} \bar{u}_{i}^{\prime \prime \prime \prime}(x)-\omega^{2} m_{i} \bar{u}_{i}(x)=0, \quad i=1,2,
$$

and $\bar{u}$ satisfies the above boundary conditions for $u$ except that $s_{2} u_{2}^{\prime \prime \prime}(l)=0$ is replaced by $s_{2} \bar{u}_{2}^{\prime \prime \prime}(l)=-\bar{Q}$. Eq. (7) gives the following sufficient condition for stationary weight:

$$
\frac{1}{c} \int_{0}^{c}\left(u_{1}^{\prime \prime} \bar{u}_{1}^{\prime \prime}-\omega^{2} b^{2} u_{1} \bar{u}_{1}\right) d x=\frac{1}{(l-c)} \int_{c}^{l}\left(u_{2}^{\prime \prime} \bar{u}_{2}^{\prime \prime}-\omega^{2} b^{2} u_{2} \bar{u}_{2}\right) d x .
$$

To find the optimal design $s_{1}, s_{2}$, Eqs. (10) and (11) are solved for $u_{i}(x)$ and $\bar{u}_{i}(x)$, respectively, in terms of $s_{1}, s_{2}$. Condition (12) and the deflection constraint $u_{2}(l)=u_{0}$ then provide two equations which yield the optimal values $s_{1}$ and $s_{2}$.

Acknowledgment. The author is indebted to Professor W. Prager for the suggestion of this topic and for helpful discussions.

\section{References}

[1] L. J. Icerman, Optimal structural design for given dynamic deflection, Internat. J. Solids \& Structures 5, 473-490 (1969)

[2] R. T. Shield and W. Prager, Optimal structural design for given deflection, Z. Angew. Math. Phys. 21, 513-523 (1970)

[3] J. M. Chern and W. Prager, Optimal design of rotating disk for given radial displacement of edge, J. Optimization Theory \& Appls. 6, 161-170 (1970)

[4] J. M. Chern, Optimal structural design for given deflection in presence of body forces, J. Optimization Theory \& Appls. (to appear)

[5] W. Prager, Optimal thermoelastic design for given deflection, Internat. J. Mech. Sci. 12, 705-709 (1970)

[6] J. M. Chern, Optimal thermoelastic design for given deformation (to appear)

[7] W. Nowacki, Dynamics of elastic systems, Wiley, New York, 1963, p. 31 